\title{
BMJ Open Protocol for a systematic review and meta-analysis of respiratory rehabilitation following intensive care unit discharge for COVID-19 survivors
}

\author{
Dan Wang (D) , ${ }^{1}$ Jin Li, ${ }^{1,2}$ Feilong Zhu (D) , Qianqin Hong, ${ }^{1}$ Ming Zhang, ${ }^{1,2}$ \\ Min Gao, ${ }^{1,2}$ Wei Chen ${ }^{2}$
}

To cite: Wang D, Li J, Zhu F, et al. Protocol for a systematic review and meta-analysis of respiratory rehabilitation following intensive care unit discharge for COVID-19 survivors. BMJ Open 2020;10:e041184. doi:10.1136/ bmjopen-2020-041184

- Prepublication history for this paper is available online. To view these files, please visit the journal online (http://dx.doi org/10.1136/bmjopen-2020041184).

Received 03 June 2020 Revised 29 0ctober 2020 Accepted 11 November 2020

A) Check for updates

(c) Author(s) (or their employer(s)) 2020. Re-use permitted under CC BY-NC. No commercial re-use. See rights and permissions. Published by BMJ.

${ }^{1}$ The Affiliated Xuzhou Rehabilitation Hospital of Xuzhou Medical University, Xuzhou Medical University, Xuzhou, Jiangsu, China

${ }^{2}$ Department of Rehabilitation Medicine, XuZhou Central Hospital, Xuzhou, Jiangsu, China

Correspondence to

Dr Wei Chen;

chenwei2339@163.com

\section{ABSTRACT}

Introduction Both physical and mental disorders may be exacerbated in patients with COVID-19 due to the experience of receiving intensive care; undergoing prolonged mechanical ventilation, sedation, proning and paralysis. Pulmonary rehabilitation is aimed to improve dyspnoea, relieve anxiety and depression, reduce the incidence of related complications, as well as prevent and improve dysfunction. However, the impact of respiratory rehabilitation on discharged patients with COVID-19 is currently unclear, especially on patients who have been mechanically ventilated over 24 hours. Therefore, we aim to investigate the efficacy of respiratory rehabilitation programmes, initiated after discharge from the intensive care unit, on the physical and mental health and healthrelated quality of life in critical patients with COVID-19.

Methods and analysis We have registered the protocol on PROSPERO and in the process of drafting it, we strictly followed the checklist of Preferred Reporting Items for Systematic Review and Meta-Analysis Potocols. We will search the PubMed, EMBASE, Web of Science, the Cochrane Central Register of Controlled Trials, China National Knowledge Infrastructure, WanFang, VIP information databases and Chinese Biomedical Literature Database. Additionally, ongoing trials in the WHO International Clinical Trials Registry Platform, ClinicalTrials.gov and ISRCTN registry will be searched as well. Studies in English or Chinese and from any country will be accepted regardless of study design. Two review authors will independently extract data and assess the quality of included studies. Continuous data are described as standard mean differences (SMDs) with $95 \%$ Cls. Dichotomous data from randomised controlled trials are described as risk ratio(RR) with 95\% Cls; otherwise, it is described as odds ratio(OR) with $95 \%$ Cls. $I^{2}$ and the Cochrane's $Q$ statistic will be used to conduct heterogeneity assessment. The quality of evidence of main outcomes will be evaluated according to the Grading of Recommendations, Assessment, Development and Evaluation(GRADE) criteria. When included studies are sufficient, we will conduct subgroup analysis and sensitivity analysis; the publication bias will be statistically analysed using a funnel plot analysis and Egger's test.

Ethics and dissemination Our review, planning to include published studies, does not need the request to the
Strengths and limitations of this study

- This review and meta-analysis is intended to provide useful recommendations for patients with COVID-19 discharged from the acute care to manage this disease from the perspective of respiratory rehabilitation.

- The findings in the future on this topic may be a critical first step in promoting and optimising the management of intensive care unit discharged adults with COVID-19.

- The main limitation may be the current lack of highlevel evidence, such as well-designed randomised controlled trials.

- These intervention studies may be frequently limited by short follow-up times and high dropout rates.

ethical committee. The final results of this review will be published in a peer-reviewed journal after completion. Patient and public involvement No patient involved. PROSPERO registration number CRD42020186791.

\section{INTRODUCTION}

In December 2019, Wuhan city, the capital of Hubei province, first witnessed the outbreak of pneumonia caused by the novel coronavirus. In March 2020, the WHO declared COVID-19 to be a pandemic. ${ }^{1}$ Although the majority of infected people develop mild or simple symptoms of COVID-19, it is estimated that approximately $14 \%$ are associated with severe respiratory tract illness and may require inpatient treatment and oxygen support. ${ }^{2}$ Furthermore, $5 \%$ of patients require intensive care. ${ }^{3}$ Patients discharged from critical care may present with chronic musculoskeletal impairments and experience profound anxiety, depression or post-traumatic stress disorder in subsequent months or years. ${ }^{4}$ This could deteriorate into delirium. ${ }^{5}$ Hence, it can be hypothesised that both physical and mental disorders may be exacerbated in patients with 
COVID-19 due to the experience of receiving intensive care; undergoing prolonged mechanical ventilation, sedation, proning and paralysis; isolation from families; rapid transfers and physical deconditioning.

According to the monthly updated systematic reviews with improvement in methodology, it is obvious that global knowledge on the needs and effects of rehabilitation against this new disease has been increasing. ${ }^{6-11}$ The recent update until August 2020 revealed that the second common limitations in functioning of rehabilitation interest $(25.5 \%)$ were respiratory structures and related functions. ${ }^{11}$ As described by two micro-level cohort studies, cardiopulmonary rehabilitation therapy is safe and feasible and effective in both acute and post-acute patients with COVID-19. ${ }^{12} 13$

There is an urgent need to provide specialist, tailored and targeted rehabilitation for COVID-19 survivors after critical care to improve health-related outcomes. Pulmonary rehabilitation is a comprehensive intervention based on a thorough patient assessment followed by patienttailored therapies that include, but not limited to, exercise training, education and behaviour change. ${ }^{14}$ It is aimed to improve dyspnoea, relieve anxiety and depression, reduce the incidence of related complications, as well as prevent and improve dysfunction. Respiratory rehabilitation delivered during hospital admission and after discharge has shown to be an effective non-drug therapy for patients with chronic respiratory diseases. ${ }^{15} 16$

However, the impact of respiratory rehabilitation on discharged patients with COVID-19 is currently unclear, especially on patients who have been mechanically ventilated for more than 24 hours. In addition, there is currently no systematic review and meta-analysis that have evaluated the effects of respiratory rehabilitation on this specific population. Therefore, we aim to investigate the efficacy of respiratory rehabilitation programmes, initiated after discharge from the intensive care unit (ICU), on the physical and mental health and health-related quality of life in critical patients with COVID-19.

\section{METHODS}

\section{Registration}

We have registered the protocol on PROSPERO. In the process of drafting the protocol, we strictly followed the checklist of Preferred Reporting Items for Systematic Review and Meta-Analysis Protocols. ${ }^{17}$

\section{Patient and public involvement}

No patient involved.

\section{Search strategy}

We intend to search the following databases: PubMed, EMBASE, Web of Science, the Cochrane Central Register of Controlled Trials, China National Knowledge Infrastructure, WanFang, VIP information databases and Chinese Biomedical Literature Database. In addition, we shall search for ongoing trials in the WHO International
Clinical Trials Registry Platform, ClinicalTrials.gov and ISRCTN registry and also explore the reference lists of included studies and contact primary authors to obtain further information regarding potentially eligible studies. Moreover, we will conduct a wider literature search of post-ICU rehabilitation including the Cochrane reviews. Studies published in English or Chinese and from any country will be included. The specific search strategy for PubMed is depicted in table 1 , for Cochrane is presented in table 2.

\section{Eligibility criteria \\ Study design}

The study design will include published randomised controlled trials (RCTs), quasi-RCTs, cluster trials, controlled clinical trials, prospective and retrospective comparative cohort studies that have compared respiratory rehabilitation after ICU discharge versus any other intervention or a control or 'usual care' programme in adults (aged $\geq 18$ years) with COVID-19 who have been mechanically ventilated for more than 24 hours, including studies presented only in abstract or letter form. We are accepting all types of trial design to enable an early comprehensive systematic review of the emerging literature.

\section{Participants}

Our study participants will be adult (aged $\geq 18$ years) patients with COVID-19 diagnosed based on positive swab test results or clinical diagnostic criteria, of any gender and race, who had been discharged from an ICU or critical care environment. The duration of ICU stay should be at least 48 hours, and the participants should have received mechanical ventilation for at least 24 hours. Patients receiving palliative care, who were pregnant or having severe comorbidities that caused their probable immobility, will be excluded.

\section{Interventions}

Chest physiotherapy includes Airway Clearance Techniques (ACTs), respiratory techniques, postural management, early mobilisation or active exercise of any duration, initiated at any time point after ICU discharge, designed to start earlier and/or to be more intensive than the care received by the control group.

We will include studies of interventions that focus solely on one or any combination of more of the following:

1. ACTs: Active Cycle of Breath Techniques, positive expiratory pressure (PEP), oscillating PEP, intermittent PEP, postural drainage, clapping, high-frequency chest wall oscillation and mechanical insufflator/exsufflator device.

2. Respiratory techniques: inspiratory or respiratory muscle training and incentive spirometers.

3. Postural management.

4. Early mobilisation: passive range of motion exercise, passive cycle ergometry, active-assisted exercises, ac- 


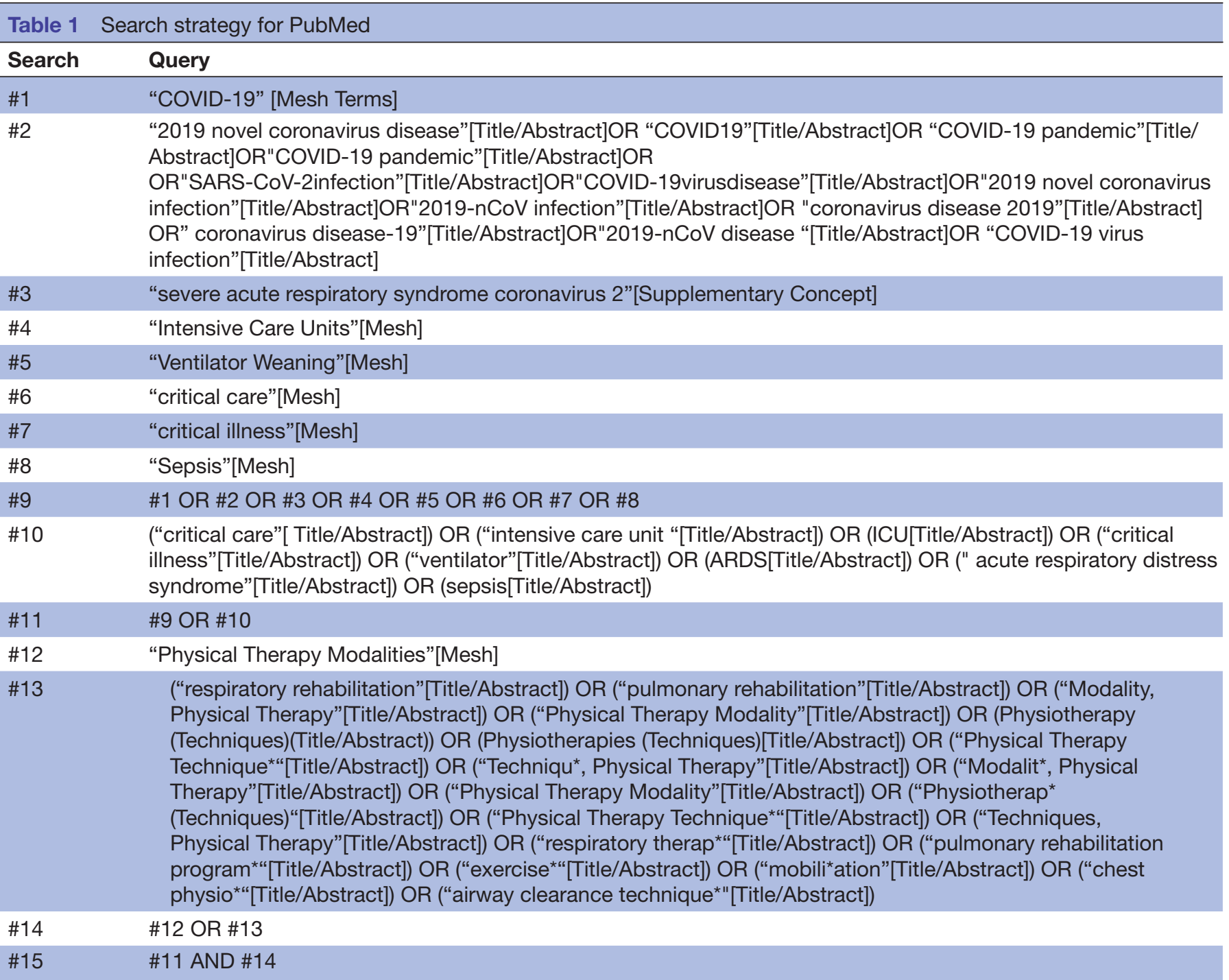

$\mathrm{MeSH}$, Medical Subject Headings.

tive range of motion exercises, activities of daily living (ADLs) and transfer training.

5. Other active exercise modes: aerobic exercise, endurance exercise, strength training, respiratory rehabilitation gymnastics, which are a type of aerobic exercise that integrates the mind, body and spirit, and theoretically based on the dynamic response of the respiratory system.

\section{Outcomes}

\section{Primary outcomes}

1. Functional exercise capacity (with physical objective assessment and/or subjective assessment): measures of physical performance, such as walking tests for example, the 6 min walk test (6MWT) and the incremental and endurance shuttle walk tests (ISWT and ESWT); or cardiopulmonary exercise test, measures of function, such as stair climbing, gait speed, stair climbing, sit-to-stand exercises and strength.

2. Pulmonary functional test: such as carbon monoxide diffusion capacity (DLCO)\%, total lung capacity
(TLC) $\%$, forced expiratory volume in the first second $\left(\mathrm{FEV}_{1}\right) \%$, forced vital capacity (FVC) $\%$ and $\mathrm{FEV}_{1} /$ FVC $\%$.

3. Adverse events (non-mortality): the risk of readmission, disability, pain, discomfort, relapses, whether expected or unexpected; or study related, possibly study-related or not study-related.

\section{Secondary outcomes}

1. Respiratory muscle function parameters measured on the body function level of the International Classification of Functioning, Disability and Health, such as maximal expiratory pressure (MEP) and maximal inspiratory pressure (MIP).

2. Mortality (defined as death at any time point during the trial duration).

3. Health-related quality of life: measured by standardised questionnaires of established reliability and validity, such as the Short-Form 36 (SF-36) (physical component score and mental component score) and the EuroQOL. 
Table 2 Search strategy for Cochrane

Search Query

$\# 1$

\#2

$\# 3$

$\# 4$

$\# 5$

\#6

\#7

\#8

$\# 9$

$\# 10$

$\# 11$

$\# 12$

$\# 13$

\#14

\#15

\#16

$\# 17$

\#18

\#19

\#20

$\# 21$

\#22

$\# 23$

\#24

$\# 25$

\#26

$\# 27$

\#28

$\# 29$

\#30

\#31

\#32

\#33

\#34

\#35

\#36

\#37

\#38

\#39

\#40

\#41

\#42

$\# 43$

\#44

\#45
MeSH descriptor: [COVID-19] explode all trees

MeSH descriptor: [critical care] explode all trees

MeSH descriptor: [intensive care unit] explode all trees

MeSH descriptor: [critical illness] explode all trees

$\mathrm{MeSH}$ descriptor: [ventilator weaning] explode all trees

MeSH descriptor: [Respiratory Distress Syndrome, Adult] explode all trees

MeSH descriptor: [Sepsis] explode all trees

\#1 OR \#2 OR \#3 OR \#4 OR \#5 OR \#6 OR \#7

"COVID-19":ti,ab,kw

"intensive care unit":ti,ab,kw

"critical care":ti,ab,kw

ICU:ti,ab,kw

"critical illness":ti,ab,kw

ventilator:ti,ab,kw

ARDS:ti,ab,kw

"acute respiratory distress syndrome":ti,ab,kw

sepsis:ti,ab,kw

\#9 OR \#10 OR \#11 OR \#12 OR \#13 OR \#14 OR \#15 OR \#16 OR \#17

\#8 OR \#18

MeSH descriptor:[Exercise]explode all trees

$\mathrm{MeSH}$ descriptor:[Exercise therapy]explode all trees

MeSH descriptor:[Rehabilitation]explode all trees

MeSH descriptor:[Physical fitness]explode all trees

MeSH descriptor:[Physical Therapy Modalities]explode all trees

\#20 OR \#21 OR \#22 OR \#23 OR \#24

exercise:ti,ab,kw

rehabilitation:ti,ab,kw

"physical fitness":ti,ab,kw

training:ti,ab,kw

mobilization:ti,ab,kw

mobilisation:ti,ab,kw

"physical therapy":ti,ab,kw

physiotherapy:ti,ab,kw

"respiratory therapy":ti,ab,kw

"electrical muscle stimulation":ti,ab,kw

"neuromuscular electrical stimulation":ti,ab,kw

"respiratory muscle training": ti,ab,kw

“inspiratory muscle training":ti,ab,kw

"cycle ergometer":ti,ab,kw

bridging:ti,ab,kw

rolling:ti,ab,kw

"lying to sitting":ti,ab,kw

marching:ti,ab,kw

ambulation:ti,ab,kw

"activities of daily living":ti,ab,kw 


\begin{tabular}{ll}
\hline Table 2 Continued & \\
\hline Search & Query \\
\hline$\# 46$ & walking:ti,ab,kw \\
$\# 47$ & \#26 OR \#27 OR \#28 OR \#29 OR \#30 OR \#31 OR \#32 OR \#33 OR \#34 OR \#35 OR \#36 OR \\
& \#37 OR \#38 OR \#39 OR \#40 OR \#41 OR \#42 OR \#43 OR \#44 OR \#45 OR \#46 \\
$\# 48$ & $\# 25$ OR \#47 \\
\#49 & \#19 AND \#48 \\
\hline
\end{tabular}

MeSH, Medical Subject Headings.

4. Activities of daily living: assess using standard measures such as the function independence measure, Barthel index scores and Katz ADLs scale.

5. Symptom assessment: modified Medical Research Committee Questionnaire; absolute Visual Analogue Scale; rating of perceived exertion scale; Borg Dyspnoea Scale.

6. Arterial blood gases and non-invasive pulse oxygen saturation: arterial oxygen partial pressure $\left(\mathrm{PaO}_{2}\right)$, oxygen saturation and oxygenation index $\left(\mathrm{PaO}_{2} /\right.$ fractional inspired oxygen).

7. Anxiety (Generalised Anxiety Disorder); depression (Patient Health Questionnaire); post-traumatic stress symptoms (Trauma Screening Questionnaire).

8. Discharge time: according to the length of hospital stay after ICU discharge.

\section{Study selection}

The databases listed above will be initially searched according to the eligibility criteria. Two review authors will independently screen these titles and abstracts, of those are not relevant will be excluded. If necessary, we will contact the authors of these studies in order to assist in the screening. Microsoft Excel will be applied to record initial decisions regarding which studies should be excluded and included, including information about database, author, title, year, journal and reasons for exclusion. We will try to obtain full-text versions of potentially relevant studies and independently demined final eligibility by joint agreement of two review authors.

For the specific process of study selection, see figure 1.

\section{Data extraction}

Two review authors will independently extract data from all selected studies using a standardised paper form that include information about study design, participants, trial characteristics, descriptions of intervention and outcomes. During this process, we will contact the primary authors of included studies if necessary. After completion of data extraction, a third reviewer will be responsible for finalising any discrepancies.

\section{Quality assessment of included studies}

Two review authors shall independently assess the risk of bias of all included studies using the new Cochrane Riskof-Bias Tool V.2.0 for RCTs and other quality assessment tools, which may be used, such as the Newcastle-Ottawa Scale for non-RCTs. ${ }^{18}$

Quality assessment of each study will be categorised as 'low risk of bias' (yes), 'uncertain risk of bias' (unclear) or 'high risk of bias' (no) for the following domains:

1. Randomisation process.

2. Deviations from intended intervention.

3. Missing outcome data.

4. Measurement of the outcome.

5. Selection of the reported result.

6. Overall.

The quality of the evidence of $6 \mathrm{MWT} / \mathrm{ISWT} / \mathrm{ESWT}$, DLCO \%, MIP, MEP and SF-36 will also be evaluated according to the Grading of Recommendations, Assessment, Development and Evaluation criteria; and classified as high, moderate, low or very low. Mortality and adverse events will be described as OR from non-RCTs or risk ratio (RR) from RCTs. We will present all the categories in the Risk of bias section.

\section{Data synthesis and statistical analysis}

Data synthesis

We will use RevMan V.5.3 to combine data. Considering the section of outcome covers several types, we would

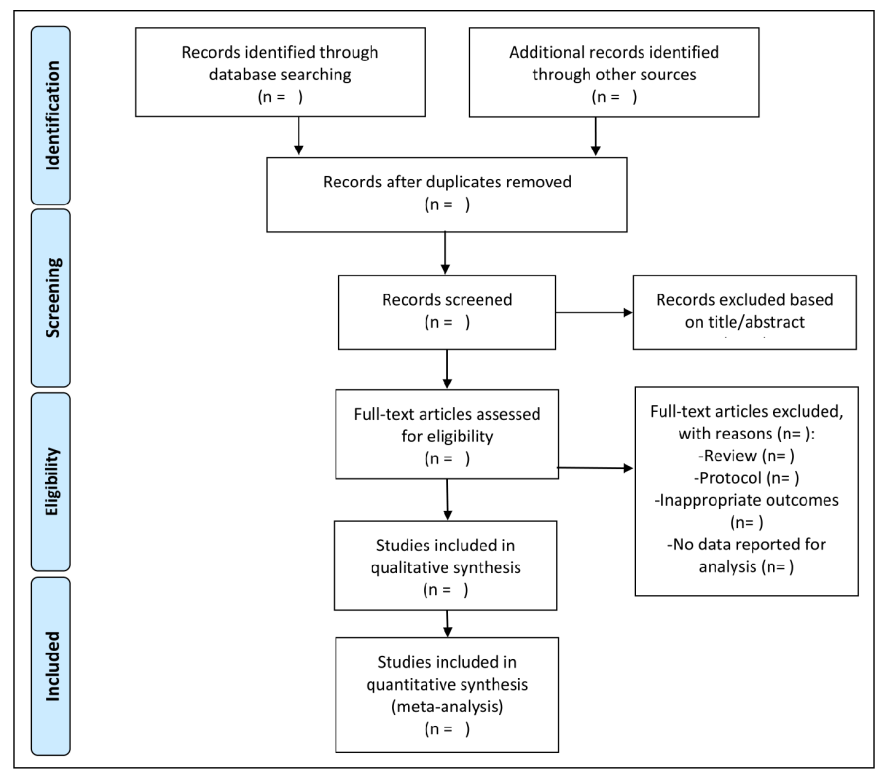

Figure 1 Preferred Reporting Items for Systematic Reviews and Meta-Analyses flow diagram. 
calculate the standardised mean difference with 95\% CIs. Dichotomous data from RCTs are described as RR with 95\% CIs; otherwise, it is described as OR with $95 \%$ CIs.

\section{Assessment of heterogeneity}

A $p$ value of $<0.1$ will be considered as statistically significant. For trials with statistically significant heterogeneity, random-effects models will be used to reduce the effects of heterogeneity between studies; otherwise, the Mantel-Haenszel fixed-effects model will be applied. The combined results will be presented using forest plots. Inconsistency among included studies will be assessed using $\mathrm{I}^{2}$ statistic with substantial heterogeneity being indicated with an $\mathrm{I}^{2}>50 \%$. We will also use the Cochrane's $Q$ statistic if it is possible to conduct a meta-analysis. ${ }^{19}$

\section{Subgroup analysis}

Subgroup analyses will be planned in the event of a sufficient number of studies, and separate analyses will be focused on rehabilitation in ICU (adults with COVID-19 who have received protocolised respiratory rehabilitation in the ICU, and patients who have not received it in the ICU), the timing of the commencement of the intervention (in hospital or after hospital discharge), the intervention duration ( 8 weeks or less, and over 8 weeks), intervention frequency (fewer than five times/ week, and five times/week or more), and type of control (no intervention and usual care). As we plan to include different trial designs, another subgroup analysis will also be conducted according to study design (ie, RCTs and non-RCTs).

\section{Sensitivity analysis}

When studies are in sufficient numbers, we intend to perform a sensitivity analysis to determine whether the estimate of effect can be affected by the level of risk of bias as follows:

1. Exclusion of studies using imputed statistics.

2. Exclusion of studies with high or unclear risk of bias.

We will also include sensitivity analysis on different interventions or complexity of interventions if possible.

\section{Assessment of publication bias}

Only when more than 10 studies show outcome data, the publication bias will be statistically analysed using a funnel plot analysis and Egger's test. The funnel plot can be used to visually measure the bias caused by the sample size.

\section{ETHICS AND DISSEMINATION}

Our review, in which we are planning to include published studies, does not require ethical committee approval. The final results of this review will be published in a peerreviewed journal after completion.

\section{DISCUSSION}

Accumulating evidence in the literature has indicated that persistent disorders of pulmonary function are a vital source of deconditioning in critical patients suffering from COVID-19. There is an increasing interest in the role of a patient-tailored rehabilitation therapy that might improve respiratory outcome in acute and postacute patients with COVID-19. ${ }^{11}$ Although the role of respiratory rehabilitation in managing patients with different chronic respiratory diseases has been scientifically confirmed, ${ }^{20}$ its effectiveness and safety when initiated after ICU discharge for adults with COVID-19 have not been established. Therefore, this review and metaanalysis is intended to provide useful recommendations for patients with COVID-19 discharged from the acute care to manage this disease from the perspective of respiratory rehabilitation.

The primary limitation might be the current lack of high-level evidence, such as well-designed RCTs. Remarkably, it may be a little early to find an adequate number of scientific papers on this topic. Therefore, we intend to extend our search protocol beyond 31 December 2020, and we shall continuously focus on the relevant scientific progress.

Contributors WC, JL and FZ designed the study. DW, FZ and QH will participate in study selection and data extraction. MZ and MG will be involved in assessment of quality. DW completed the protocol manuscript. All authors read and approved the final version of the manuscript as submitted.

Funding This study was supported by grants from Xuzhou Science \& Technology Bureau Project (KC18184) and Jiangsu Provincial Health and Family Planning Commission Project (QNRC2016376).

Competing interests None declared.

Patient and public involvement Patients and/or the public were not involved in the design, or conduct, or reporting, or dissemination plans of this research.

Patient consent for publication Not required.

Provenance and peer review Not commissioned; externally peer reviewed.

Open access This is an open access article distributed in accordance with the Creative Commons Attribution Non Commercial (CC BY-NC 4.0) license, which permits others to distribute, remix, adapt, build upon this work non-commercially, and license their derivative works on different terms, provided the original work is properly cited, appropriate credit is given, any changes made indicated, and the use is non-commercial. See: http://creativecommons.org/licenses/by-nc/4.0/.

\section{ORCID iDs}

Dan Wang http://orcid.org/0000-0002-4688-8215

Feilong Zhu http://orcid.org/0000-0001-5404-8745

\section{REFERENCES}

1 WHO. Coronavirus disease (COVID-2019) situation reports, 2020. Available: https://www.who.int/emergencies/diseases/novelcoronavirus-2019/situation-reports [Accessed May 28 2020].

2 Epidemiology Working Group for NCIP Epidemic Response, Chinese Center for Disease Control and Prevention. [The epidemiological characteristics of an outbreak of 2019 novel coronavirus diseases (COVID-19) in China]. Zhonghua Liu Xing Bing Xue Za Zhi 2020;41:145-51.

3 World Health Organization. Clinical management of severe acute respiratory infection (SARI) when COVID-19 disease is suspected. interim guidance, v1.2. 2020.

4 Wade DM, Howell DC, Weinman JA, et al. Investigating risk factors for psychological morbidity three months after intensive care: a prospective cohort study. Crit Care 2012;16:R192.

5 Wade DM, Brewin CR, Howell DCJ, et al. Intrusive memories of hallucinations and delusions in traumatized intensive care patients: an interview study. Br J Health Psychol 2015;20:613-31. 
6 Ceravolo MG, de Sire A, Andrenelli E, et al. Systematic rapid "living" review on rehabilitation needs due to COVID-19: update to March 31st, 2020. Eur J Phys Rehabil Med 2020;56:347-53.

7 de Sire A, Andrenelli E, Negrini F, et al. Systematic rapid living review on rehabilitation needs due to COVID-19: update as of April 30th, 2020. Eur J Phys Rehabil Med 2020;56:354-60.

8 Ceravolo MG, de Sire A, Andrenelli E, et al. Systematic rapid "living" review on rehabilitation needs due to COVID-19: update to March 31st, 2020. Eur J Phys Rehabil Med 2020;56:347-53.

9 Ceravolo MG, Arienti C, de Sire A, et al. Rehabilitation and COVID-19: the Cochrane rehabilitation 2020 rapid living systematic review. Eur J Phys Rehabil Med 2020;56:642-51.

10 Negrini F, de Sire A, Andrenelli E, et al. Rehabilitation and COVID-19: the Cochrane rehabilitation 2020 rapid living systematic review. update as of July 31st, 2020. Eur J Phys Rehabil Med 2020;56:652-7.

11 De Sire A, Andrenelli E, Negrini F, et al. Rehabilitation and COVID-19: the Cochrane rehabilitation 2020 rapid living systematic review. update as of August 31st, 2020. Eur J Phys Rehabil Med 2020. doi:10.23736/ S1973-9087.20.06614-9. [Epub ahead of print: 0110 2020].

12 Hermann M, Pekacka-Egli A-M, Witassek F, et al. Feasibility and efficacy of cardiopulmonary rehabilitation after COVID-19. Am J Phys Med Rehabil 2020;99:865-9.

13 Zha L, Xu X, Wang D, et al. Modified rehabilitation exercises for mild cases of COVID-19. Ann Palliat Med 2020;9:3100-6.
14 Rochester CL, Vogiatzis I, Holland AE, et al. An official American thoracic Society/European respiratory Society policy statement: enhancing implementation, use, and delivery of pulmonary rehabilitation. Am J Respir Crit Care Med 2015;192:1373-86.

15 Puhan MA, Gimeno-Santos E, Cates CJ, et al. Pulmonary rehabilitation following exacerbations of chronic obstructive pulmonary disease. Cochrane Database Syst Rev 2016;12:CD005305

16 Puhan MA, Scharplatz M, Troosters T, et al. Respiratory rehabilitation after acute exacerbation of COPD may reduce risk for readmission and mortality - a systematic review. Respir Res 2005;6:54.

17 Moher D, Shamseer L, Clarke M, et al. Preferred reporting items for systematic review and meta-analysis protocols (PRISMA-P) 2015 statement. Syst Rev 2015;4:1.

18 Higgins JPT, Thomas J, Chandler J, eds. Cochrane Handbook for Systematic Reviews of Interventions. 2nd ed. Chichester (UK): John Wiley \& Sons, 2019.

19 Moher D, Liberati A, Tetzlaff J, et al. Preferred reporting items for systematic reviews and meta-analyses: the PRISMA statement. PLoS Med 2009;6:e1000097.

20 Gloeckl R, Schneeberger T, Jarosch I, et al. Pulmonary rehabilitation and exercise training in chronic obstructive pulmonary disease. Dtsch Arztebl Int 2018;115:117-23. 